\title{
Long-Term Monitoring of Intracardiac Pressure at Home using a Wireless Implantable Sensor
}

\author{
Henrik Casimir Ahn, MD, $\mathrm{PhD}^{1^{*}}$ and Jacek Baranowski, MD, $\mathrm{PhD}^{2}$ \\ ${ }^{1}$ Department of Cardiothoracic Surgery, Institution of Medical and Health Sciences, Linkoping University, Linkoping, Sweden \\ ${ }^{2}$ Department of Physiology, University Hospital, Linkoping, Sweden
}

"Corresponding author: Prof Henrik Casimir Ahn, Department of Cardiothoracic Surgery, Institution of Medical and Health Sciences, Linkoping University, Linkoping, Sweden, Tel: + 46707256463; E-mail: henrik.casimir.ahn@liu.se

Received: December 12, 2018; Accepted: January 02, 2019; Published: January 07, 2019

Copyright: @ 2019 Ahn HC, et al. This is an open-access article distributed under the terms of the creative commons attribution license, which permits unrestricted use, distribution, and reproduction in any medium, provided the original author and source are credited.

\begin{abstract}
Background: We have previously reported results from an initial study on a new implantable intracardiac wireless hemodynamic monitor in man, showing favourable results regarding accuracy of recordings over time and safety profile. The aim of this study was to follow up the intervention group of 16 patients with left atrial implants over a longer period to determine the systems longevity and dependability.
\end{abstract}

Material: The $\operatorname{Titan}^{\mathrm{TM}}$ monitoring system using a miniature pressure sensor was implanted in 16 patients undergoing open chest cardiac surgery. After discharge the system was used for left atrial pressure monitoring at home.

Findings: No major or minor adverse events were observed. As of July 2018, the average age of functioning left atrial implants providing high-quality hemodynamic curves was 3.75 years, ranging from 3.25 years to 5 years.

Conclusion: The system was reliable, provided high-quality pressure curves and was easy to use. Recalibration using the non-invasive Valsalva maneuver was accurate and easy to perform.

Keywords: Heart failure; Diagnostics; Pressure monitoring; Telemedicine

\section{Introduction}

The possibility of monitoring heart function more accurately in heart failure patients has gained increasing interest. The ability to detect a sudden increase in filling pressure would provide early warning of an imminent exacerbation and enable medical intervention before the development of clinical symptoms [1]. A catheter-based technology with a device introduced into the pulmonary artery allowing wireless recordings of pulmonary artery pressure has been approved by the Food and Drug Administration [2,3].

We have previously reported results from an initial study on an implantable intracardiac wireless pressure sensor using a MEMS (Micro Electro Mechanical Systems) pressure-sensing element with custom electronics (Titan ${ }^{\mathrm{Tn}}$ ) in man. This technology has previously been shown to enable direct measurement of intracardiac pressures with curve patterns similar to those achieved by a Millar reference catheter or a standard fluid-filled catheter [4-6]. In a previous study [6], the device was implanted in a series of 40 patients with heart failure during a 2-year period from June 2013 until June 2015. Thirtyone patients scheduled for open heart surgery and 9 for transcatheter aortic valve replacement (TAVR) were included to test the safety and feasibility of the $\operatorname{Titan}^{\mathrm{T}}$ pressure sensor. After written consent the patients were randomized to the implant or control group (20/20 patients). The baseline data have been reported elsewhere [6]. The pressure sensor provided accurate, rapid, detailed continuous real-time pressure measurements with a good safety profile [6]. The aim of this follow-up study was to see if the pressure sensor continues to give accurate and reproducible intracardiac pressure recordings over longer periods of time. Since this report focuses on function of the implants beyond the initial study period, data from the control group were not considered.

\section{Materials and Methods}

This study was approved by the Swedish Medical Products Agency Dnr: 461:2012/518610 and the Regional Ethics Committee Dnr: 2013/50-31. Recordings were observed until July 1, 2018. At the initial operation, 16 patients had a sensor placed in the left atrium (LA) and 4 in the left ventricle (LV). Only $2 \mathrm{LV}$ patients were alive June 2018; 1 had stopped taking measurements the year before, leaving 1 patient performing ongoing recordings. For this reason, data from the LV patients were not considered in this follow-up study. Of the remaining 16 patients with a LA sensor, 15 were still alive June 2018. Patient characteristics are shown in Table 1.

\section{Device description}

The TitanTM Wireless Implantable Hemodynamic Monitoring (WIHM) System uses a miniature, on-demand, wireless pressure sensor that is implanted in the left atrium or left ventricle as an adjunct device during open-chest surgery. The WIHM System utilizes highfrequency radiofrequency (RF) energy to power the sensor and to measure left atrial pressure. The system consists of three parts:

1) A miniature implantable pressure sensor without an implanted power source such as a battery or wires (Figure 1). 
Citation: Ahn HC, Baranowski J (2019) Long-Term Monitoring of Intracardiac Pressure at Home using a Wireless Implantable Sensor. J Cardiovasc Dis Diagn 7: 354. doi:10.4172/2329-9517.1000354

Page 2 of 5

2) A monitoring unit with readout electronics, antenna and user interface for monitoring both at home and in the hospital (Figure 2).

3) An internet-mediated communication system and central server with an integrated information security system.

\begin{tabular}{|l|l|}
\hline Patient (n=16) characteristics & $\begin{array}{l}\text { Patients with LA } \\
\text { sensor implant }\end{array}$ \\
\hline Age, mean + range at follow up & $73(63-89)$ years \\
\hline Gender (M/F) & $11 / 5 a$ \\
\hline NYHA (III/IV) & $13 / 3 a$ \\
\hline Procedure AVR/MI-repair/ CABG & $5 / 7 / 4 a$ \\
\hline On warfarin & 10 \\
\hline On antiplatelet therapy & 6 \\
\hline
\end{tabular}

M: Male, F: Female, NYHA: New York Heart Association, MI: Mitral Insufficiency, CAD: Coronary Artery Disease, AVR: Aortic Valve Replacement, CABG: Coronary Artery Bypass Grafting, LA: Left Atrium.

Table 1: Patient characteristics.

The telemetric pressure sensor consists of a cylindrical pressuresensing probe placed inside a polyether ketone polymer anchor (Figure 1). The probe contains a MEMS (Micro-Electro- Mechanical System) pressure-sensing element along with custom electronics. The sensing element is located at the end of the cylindrical implant that projects into the left atrial or left ventricle chamber. All sensor components are made of materials that have been chosen for their biocompatibility, durability and insensitivity to changes in body chemistry or biology $[4,5]$. The sensor uses passive electronics to transduce and transmit pressure signals. Power is supplied wirelessly via RF telemetry without the need for a battery. The power consumption of the sensor is very low ( $<50$ microwatts) and there is thus no risk for the patient or pain during measurement or recording.

The antenna (Figure 2) sends a signal and provides power to the sensor, which in turn sends a signal back to the antenna that carries the pressure data. The RF communication is achieved using power that is many times smaller than commercial RF scanners and is within established guidelines, including those of the FDA and FCC.

This modulated signal is converted into a digital signal by the monitoring unit (MU). This is wirelessly transmitted to a database through a custom graphical user interface-based application program running on the MU that provides the user interface (Figure 3). The application has been developed in the LABVIEWTM graphical programming environment.

\section{Surgical procedure}

The sensor was permanently implanted in the left atrium (LA) of the 16 patients at the end of an open-chest cardiac procedure. The sensor was located at the border between the right superior pulmonary vein and the roof of the LA and fixed to the epicardium by sutures through preformed holes in the proximal flange of the sensor.

\section{Home monitoring}

After instruction prior to discharge from the hospital, the patients were able to take pressure measurements at home using the hand-held antenna and monitor. The system was installed in the home by a medical engineer and connected to a local network.

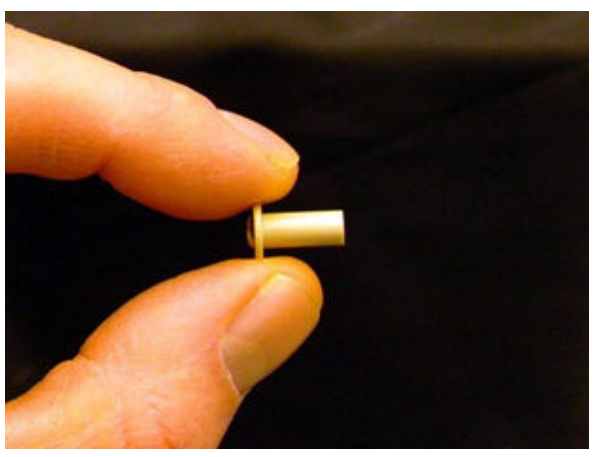

Figure 1: The TitanTM telemetric sensor.

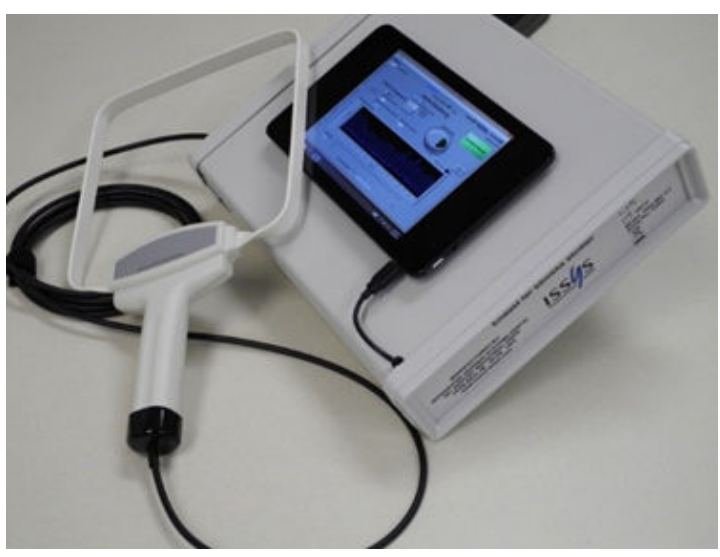

Figure 2: The $\operatorname{Titan}^{\mathrm{Tm}}$ monitoring unit.

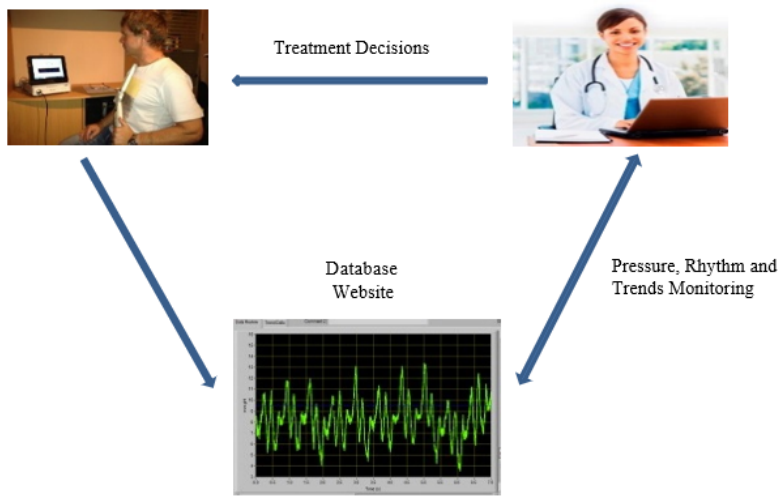

Figure 3: $\operatorname{Titan}^{\text {Tx }}$ patient database.

The patient was required to push one button to take measurements and record 10 seconds of continuous pressure readings to provide a curve. The results were then transmitted via Internet to a remote 
database (Figure 4). The data could be accessed at any time via the internet by the physician responsible or a nurse (Figure 3 ).

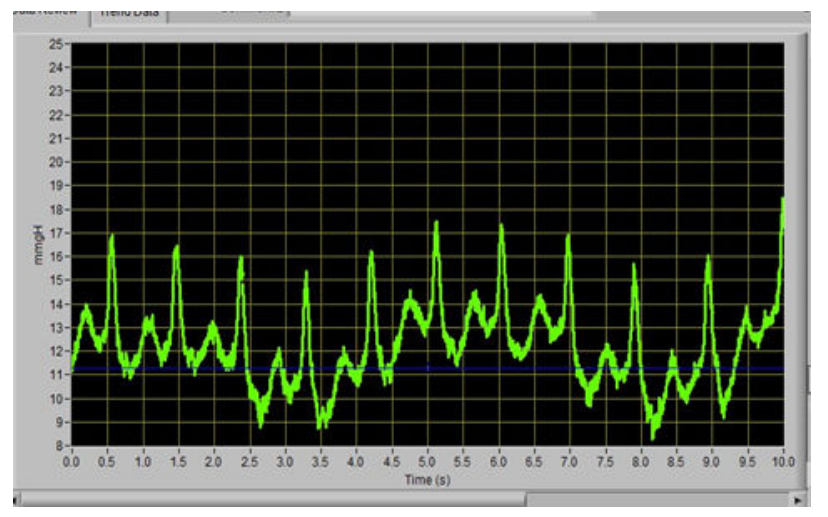

Figure 4: A typical curve from a recording at home.

\section{Development}

In order to compensate for drift over time, the company developed a non-invasive calibration technique based on the Valsalva maneuver (VM). The patient takes a deep breath and then forces expiration against an obstructing airway pressure monitor producing a high intrathoracic pressure. After 3-5 seconds the pressure in the airway equilibrates with the left heart filling pressure (i.e., mean LA pressure or left ventricle end diastolic pressure). Calibration is performed by simultaneously measuring the airway pressure through the monitoring unit and the left heart filling pressure recorded by the sensor. This procedure allows any offset to be measured and easily incorporated into the patient's monitoring program.

The medical investigators were easily able to perform VM calibration in all cases, and this simple procedure-maintained function and accuracy of the sensor. All LA implants (15/16) provided high fidelity waveforms with high-quality pressure curves. Nineteen patients were able to use the $\operatorname{Titan}^{\text {Th }}$ monitor unit. Patient \#3, a woman then aged 84 years, operated Oct. 10, 2013 was not able to do the measurements by herself and was initially helped by a nurse. At the age of 88 , however, she managed to carry out Valsalva calibration. Regular transthoracic echocardiography and the occasional transoesophageal echocardiography were performed, neither of these showing any evidence of tissue growth around the sensor. The implant was clearly visible during these investigations indicating that no significant tissue overgrowth of the sensor occurred even after 3-4 years in use.

\section{Statistical Analysis}

The data were prospectively stored in the hospital database and transferred to an Excel database for analysis. Endpoints were: 1) the ability to achieve a measurable sensor signal after more than 2 years of use; 2) if a signal is obtained how the adequacy and reproducibility of the pressure recordings is. The null hypotheses were: a) the sensor does not give a measurable signal after 2 years and b) even if a signal is obtained, a physiological curve and pressure values within the expected range cannot be achieved. Categorical variables "yes" and "no" after the last recording registered for each patient were compared using the chi-square test. A p value $<0.05$ between the groups was regarded as significant. Statistica (StatSoft Inc.) software was used for the calculations.

\section{Results}

The long-term data for the implant patient cohort are shown in Table 2. The patients are listed in the order they were included in the original study corresponding to the original clinical report form (CRF) for each patient with a 2-year follow-up. Patient \#2 was the second of two pilot patients who followed the same study protocol. He is included in the present study because he was the one of the surviving patients who had had the implant for the longest time. Patient \#41 was included to replace a patient who was initially randomized to the sensor group but was excluded at the operation.

In Patient \#36, the LA signal disappeared within 3 months and never returned; probably due to equipment failure [6]. The patient deteriorated successively and died of severe heart failure 3 years after surgery. Two patients stopped home monitoring after $3 \frac{1 / 2}{2}$ years for personal reasons, but not due to implant failure. Patient \#3 had no experience of computer devices prior to the study and was the only patient who could not cope with the equipment. Instead, a nurse helped her with recordings. She eventually became very frail and we decided to terminate her participation in the follow-up. Patient \#8 stopped taking recordings after $3^{1 / 2}$ years due to recurrent problems with his local network and internet connection. Patient \#29 had initial heart failure symptoms after the operation, with rising LA-pressures due to pericardial effusion and imminent tamponade. She was reoperated with decompression of the heart and removal of blood clots. She developed a longstanding inflammatory reaction with pericardial effusion that successively declined after treatment. The sensor provided unstable recordings for a while but then stabilized within the expected range. Eventually, however, it drifted to levels that eventually made readings unreliable. All remaining patients have successfully developed routines for making intermittent recordings.

Between May and July 2017, all patients were paid a visit to upgrade their system software. Calibrations were made with the patient sitting in a chair and performing the Valsalva maneuver. All patients but one managed this short respiratory maneuver while the output signal was being monitored. Pt \#36 did not participate due to implant failure. The final collection of data for this report was completed July 2018. Thirteen of the 16 survivors had recent recording data for analysis. Time after surgery and the most recent pressure values are shown in Table 2 (columns 7-9). When calculating the total time for each patient and the average time after surgery, Patients \#3 and \# 8 were included using data from the period up until they stopped taking measurements. The average time as of July 2018 was 3.75 years months, ranging 3.25 - 5 years.

All patients were on anticoagulation or antiplatelet therapy during follow-up, depending on the procedure they underwent. Three patients developed curve patterns showing atrial fibrillation (AF) during follow-up without symptoms at that time. They were initially on low-dose aspirin only, but once AF was confirmed they were put on anticoagulation prior to electro conversion. Since it was possible to detect an LA sensor signal that provided easily measurable, physiologically adequate pressure values after an average implantation time of 3.75 years in 15 of 16 patients, the null hypotheses were rejected. 


\begin{tabular}{|c|c|c|c|c|c|c|c|}
\hline $\begin{array}{l}\text { Patient Study } \\
\#\end{array}$ & $\begin{array}{l}\text { Date of operation } \\
\text { (month, year) }\end{array}$ & $\begin{array}{l}\text { Age, Gender } \\
\text { (M/F) }\end{array}$ & $\begin{array}{l}\text { Sensor curve } \\
\text { and signal at } 6 \\
\text { months }\end{array}$ & $\begin{array}{l}\text { Recording with } \\
\text { Valsalva maneuver }\end{array}$ & $\begin{array}{l}\text { Date of last } \\
\text { Recording }\end{array}$ & $\begin{array}{l}\text { Sensor curve } \\
\text { and signal at } \\
\text { (Number } \\
\text { years, months) }\end{array}$ & $\begin{array}{l}\text { LA pressure, } \mathrm{mm} \\
\mathrm{Hg}\end{array}$ \\
\hline 2 & Jun-13 & M, 63 & Yes & Jun-17 & Jun-18 & 5 & 7 \\
\hline 3 & Oct-13 & $F, 89$ & Yes & May-17 & May-17 & 3,7 & 12 \\
\hline 8 & Nov-13 & M, 69 & Yes & Jul-17 & Jul-17 & 3,8 & 10 \\
\hline 11 & Jan-14 & M, 78 & Yes & Jul-17 & Feb-18 & 4,1 & 11 \\
\hline 17 & Apr-14 & $\mathrm{F}, 66$ & Yes & Jun-17 & Mar-18 & 3,11 & 10 \\
\hline 19 & May-14 & $F, 78$ & Yes & May-17 & Mar-18 & 3,10 & 20 \\
\hline 21 & Jun-14 & $\mathrm{F}, 66$ & Yes & Jun-17 & Oct-17 & 3,4 & 14 \\
\hline 22 & Jun-14 & M, 73 & Yes & May-17 & Nov-17 & 3,6 & 3 \\
\hline 28 & Oct-14 & M, 78 & Yes & May-17 & Apr-18 & 4,1 & 17 \\
\hline 29 & Oct-14 & $\mathrm{F}, 74$ & Yes & May-17 & Feb-18 & 3,5 & 65 \\
\hline 32 & Oct-14 & M, 68 & Yes & May-17 & Nov-17 & 3,1 & 16 \\
\hline 34 & Nov-14 & M, 67 & Yes & May-17 & Apr-18 & 3,5 & 10 \\
\hline 36 & Nov-14 & M, 76 & No & NA & NA & NA & NA \\
\hline 37 & Nov-14 & M, 81 & Yes & Jun-17 & Apr-18 & 3,4 & 8 \\
\hline 39 & Dec-14 & M, 76 & Yes & May-17 & Apr-18 & 3,4 & 16 \\
\hline 41 & Jan-15 & M, 72 & Yes & Jun-17 & Jun-18 & 3,5 & 17 \\
\hline
\end{tabular}

Table 2: All patients in chronological order who received a left atrial implant at the time of open-heart surgery. The columns contain data from 6 months follow-up (column 4), Valsalva maneuver calibration date with pressure measurements (column 5), and the last date for patient home measurement before data collection for this report (columns 6).

\section{Discussion}

Heart failure is a huge healthcare problem. The disease may be stable in the initial stages, but progressive deterioration develops with exacerbations that initially respond to standard medical treatment. Eventually, symptoms of heart failure become more and more predominant. The course is often unpredictable and in the final stage's exacerbations lead to recurrent hospitalization. An exacerbation may be associated with and even preceded by increased filling pressures in the heart. Home monitoring of pulmonary artery pressure with a wireless pressure sensor has been shown to reduce the number of hospitalizations of patients with heart failure, despite the fact that pulmonary pressure is not directly related to true intracardiac pressure $[2,3]$.

The miniature wireless pressure sensor used in the present study enables direct measurement of intracardiac pressures with curve patterns similar to those achieved by a Millar reference catheter or a standard fluid-filled catheter [4-6]. Echocardiographic investigations were carried out after 1, 3, 6 and 24 months, as part of our first human study, for all patients with the current implant [6]. The implant could be visualized by echocardiography in all cases, and no observations of thrombotic material or tissue overgrowth were reported. Besides the recording problems mentioned previously, no clinically adverse event associated with the implant was seen. We extended the follow-up of our sensor patients because the implants continued to provide good signals that, after simple non-invasive Valsalva calibration and software update, gave reliable and reproducible pressure values in the expected range. After almost five years in use, our first patient had a curve pattern identical to the initial postoperative recording and with a comparable mean LA pressure. The overall results after an average follow-up time of more than $3 \frac{1}{2}$ years indicate good biocompatibility and functional life of the LA implants with no significant tissue overgrowth.

One unexpected, and highly beneficial, spin-off of home monitoring was the rapid detection of arrhythmias such as atrial fibrillation and flutter, despite absence of symptoms, when the patient observed a change in their curve pattern. Further analysis of the clinical data is beyond the scope of this study but will be reported separately in a comparison with the control group that was included in the original prospective randomized study [7-9]. The pressure sensor used in this study has been used in association with implantation of a left ventricular assist device [10]. Monitoring of LA pressure is often used in the postoperative course for optimal regulation of pump speed. We performed concomitant LA pressure measurement and echocardiographic assessment of left ventricular end diastolic dimension at different pump speeds and found an excellent correlation 
Citation: Ahn HC, Baranowski J (2019) Long-Term Monitoring of Intracardiac Pressure at Home using a Wireless Implantable Sensor. J Cardiovasc Dis Diagn 7: 354. doi:10.4172/2329-9517.1000354

Page 5 of 5

between these variables [10,11]. Theoretically, feedback from a pressure transducer to the pump speed regulator could provide a means of matching pump speed to physiologic demands. The sensor implant in this follow up study was inserted on completion of open chest surgery. A slightly modified implant for catheter-based insertion is currently under investigation in our animal lab, and eventually we plan to test this in a human feasibility study.

\section{Conclusion}

A miniature wireless intracardiac pressure sensor was shown to be safe and easy to use. Home monitoring using left atrial implants has so far functioned well; 3.75 years on average and, in some cases, between 4 and 5 years without adverse advents. The recordings provide highquality pressure curves that are reliable and reproducible over time. A non-invasive Valsalva maneuver-based calibration procedure was found to be feasible for testing the accuracy of the pressure values and recalibration of the monitor when necessary.

\section{Conflicts of Interest}

There are no conflicts of interest for the present study.

\section{References}

1. Smith SA, Abraham WT (2012) Implantable cardiovascular sensors and computers: Interventional heart failure strategies. Curr Cardiol Rep 14: 611-618.

2. Abraham WT, Adamson PB, Bourge RC, Aaron MF, Costanzo MR, et al. (2011) Wireless pulmonary artery haemodynamic monitoring in chronic heart failure: A randomised controlled trial. Lancet 377: 658-666.
3. Loh JP, Barbash IM, Waksman R (2013) Overview of the 2011 Food and Drug Administration circulatory system devices panel of the medical devices advisory committee meeting on the CardioMEMS champion heart failure monitoring system. J Am Coll Cardiol 61: 1571-1576.

4. Najafi N, Ludomirsky A (2004) Initial animal studies of a wireless, batteryless, MEMS implant for cardiovascular applications. Biomed Microdevices 6: 61-65.

5. Hammond RL, Hanna K, Morgan C, Perakis P, Najafi N, et al. (2012) A wireless and battery-less miniature intracardiac pressure sensor: Early implantation studies. Asaio J 58: 83-87.

6. Ahn HC, Delshad B, Baranowski J (2016) An implantable pressure sensor for long-term wireless monitoring of cardiac function - First study in man. J Cardiovasc Dis Diagn 4: 252.

7. Bourge RC, Abraham WT, Adamson PB, Aaron MF, Aranda JM, et al (2008) Randomized controlled trial of an implantable continuous hemodynamic monitor in patients with advanced heart failure: The COMPASS-HF study. J Am Coll Cardiol 51: 1073-1079.

8. Ritzema J, Troughton R, Melton I, Crozier I, Doughty R, et al. (2010) Hemodynamically Guided Home Self-Therapy in Severe Heart Failure Patients (HOMEOSTASIS) Study Group. Physician directed patient selfmanagement of left atrial pressure in advanced chronic heart failure. Circulation 121: 1086-1095.

9. Troughton RW, Ritzema J, Eigler NL, Melton IC, Krum H, et al. (2011) Direct left atrial pressure monitoring in severe heart failure: Long-term sensor performance. J Cardiovasc Transl Res 4: 3-13.

10. Hubbert L, Baranowski J, Delshad B, Ahn H (2017) Left atrial pressure monitoring with an implantable wireless pressure sensor after implantation of a left ventricular assist device. Asaio J 63: e60-65.

11. McClean D, Aragon J, Jamali A, Kar S, Ritzema-Carter J, et al. (2006) Non-invasive calibration of cardiac pressure transducers in patients with heart failure: An aid to implantable hemodynamic monitoring and therapeutic guidance. J Card Fail 12: 568-576. 